\title{
Studies on the formation of O/W nano-emulsions, by low-energy emulsification method, suitable for cosmeceutical applications*
}

\author{
Małgorzata Jaworska1凶, Elżbieta Sikora1, Michał Zielina² and Jan Ogonowski \\ ${ }^{1}$ Faculty of Chemical Engineering and Technology, Institute of Organic Chemistry and Technology, Cracow University of Technology, Kraków, Po- \\ land; 2Faculty of Environmental Engineering, Institute of Water Supply and Environmental Protection, Cracow University of Technology, Kraków, \\ Poland
}

The formation of oil/water (O/W) nano-emulsions suitable for cosmeceutical application was studied. Nanoemulsions were prepared by using phase inversion composition (PIC) method, one of the low-energy emulsification methods. The process consist of stepwise water addition to oil/surfactant mixture, at $\mathrm{T}=25^{\circ} \mathrm{C}$. Caprylic/ capric triglycerides (GTCC), propylene glycol dicaprylate/ dicaprate $(\mathrm{PC})$ and oleic acid $(\mathrm{OA})$ were applied as an oil phase. Polysorbate $\mathbf{8 0}$ was used as the surfactant. Kinetic stability of the nano-emulsions was analyzed by measuring droplet size as a function of time for different oil/surfactant ratio. The particles size distribution was analyzed by means DLS measurement technique (Dynamic Light Scattering), using Zetasizer Nano ZS (Malvern Instruments, UK). One of triterpenoic acid, practically non-water soluble substance was selected as an active and incorporated into the stable formulation. The obtained results proved that the nanoemulsion NE-T80-GTCC-20:80 based on caprylic/capric triglycerides with the oil/surfactant ratio $O / S=2 \quad 0: 80$ and the droplet size $r=25 \mathrm{~nm}$ was the most stable one and additionally showed the highest solubilisation capacity for the triterpene.

Key words: nano-emulsions, crodamol GTCC, crodamol PC, oleic acid

Received: 15 October, 2013; revised: 04 December, 2013; accepted: 04 December, 2013; available on-line: 29 December, 2013

\section{INTRODUCTION}

Cosmeceuticals are cosmetic products with biologically active ingredients, showing not only cosmetic but also drug-like properties, used for the care and the improvement of skin conditions (Gao et al., 2008).

Nano-emulsions are one of the most promising system for transdermal delivery of active substances because of the ease of preparation, long term stability, absence of organic solvents and high solubilization capacity for both, hydrophilic and lipophilicactives (Maghraby, 2008; Peltola et al., 2003). Moreover, nano-sized systems improve the penetration of active ingredients into the skin (Teo et al., 2010). Apart from good cosmetic properties also from technological point of view the nano-emulsions offer several advantages for cosmeceutical application. Due to the small droplets sizes, they are characterized by kinetic stability against sedimentation, flocculation and coalescence. It also helps to resist the process of creaming because the droplet's Brownian motion overcoming gravity force (Tadros et al., 2004).
Currently, nano-emulsions are object of interest to pharmaceutical manufacturers as SEDDS (self-emulsifying drug delivery systems), an isotropic mixtures of oils, surfactants, solvents and co-solvents/surfactants which can be used for the design of formulations in order to improve the oral absorption of highly lipophilic drug compounds (Gursoy et al., 2004). Thanks to their properties it was possible to increase absorption after oral administration of poorly water-soluble drugs: Sandimmune ${ }^{\circledR}$ and Sandimmun Neoral ${ }^{\circledR}$ (cyclosporin A), Norvir $^{\circledR}$ (ritonavir), and Fortovase ${ }^{\circledR}$ (saquinavir) (Gursoy et al., 2004), Sporanox ${ }^{\circledR}$ (itraconazole) ( Hong et al., 2006), Coenzyme Q10 (Balakrishnan et al., 2009) or proteins such as $\beta$-lactamase (Rao et al. 2008).

Terpenoids are the largest group of natural bioactive compounds exhibiting multiple nutriceutical activities, they are also known as active cosmetic ingredients (Wang et al., 2005). Especially the triterpene acids, (e.g. betulinic, maslinic, oleanolic, ursolic) exhibit unique biological and pharmacological activities such as: anti-inflammatory, antimicrobial, antiviral properties, cytotoxic effects, against cancer and cardiovascular diseases (Silva et al. 2012).

The formation of oil/water $(\mathrm{O} / \mathrm{W})$ nano-emulsions as a vehicle for triterpene acid, suitable for cosmeceutical application was studied.

\section{MATERIAL AND METHODS}

Non-ionic surfactant, Tween ${ }^{\circledR} 80$ (INCI: Polysorbate 80) was obtained from Sigma-Aldrich. The surfactant was selected because of its good cosmetic properties and lack of irritation and toxicity to the skin. The oils used in the study: Crodamol GTCC ${ }^{\circledR}$ (INCI: Caprylic/Capric Trigliceryde, $H L B_{R}=9.88$ ), Crodamol PC (Propylene Glycol Dicaprylate/Dicaprate, $\left.H L B_{R}=10.99\right)$ were purchased from Croda Poland. Oleic Acid $\left(H L B_{R}=16.00\right)$ was supplied by Chempur. As the aqueous phase of the nano-emulsions distilled water was used.

Nano-emulsions were prepared by stepwise water (W) addition to the mixture of oil (O) and surfactant (S), at room temperature $\left(25^{\circ} \mathrm{C}\right)$. The mixture was homogenized using IKA VORTEX GENIUS 3. The compositions were considered as nano-emulsions when they

e-mail: mjaworska@chemia.pk.edu.pl

* Presented at the 5th Central European Congress of Life Sciences EUROBIOTECH 2013

Abbreviations: GTCC, caprylic/capric triglycerides; OA, oleic acid; $\mathrm{O}$, oil; O/S, oil/surfactant; O/W, oil/water; PC, propylene glycol dicaprylate/dicaprate; S, surfactant; W, water 
Table 1. Composition and characteristic of the formulations.

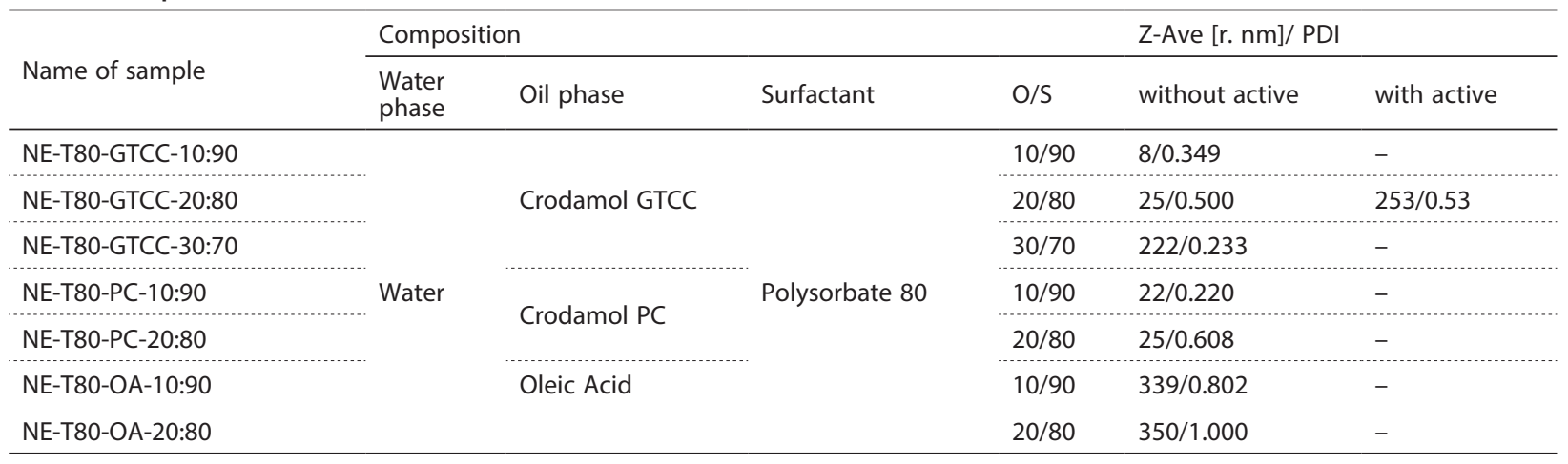

were transparent and translucent, showing a bluish shine or opaque. It was confirmed that they were not microemulsions as their properties depended on the preparation method and the storage temperature.

The mean droplet sizes of nano-emulsions were measured by Dynamic Light Scattering (DLS) method, using Malvern Zetasizer Nano ZS apparatus, which measures particle size (range from $0.3 \mathrm{~nm}$ to $10 \mu \mathrm{m}$ ) by scattering photons from a sample and determine the change in diffracted light intensity. Scattering angle was $173^{\circ}$. The analysis was performed three times for each sample to determine mean values.

The emulsions stability was assessed by measuring droplet size as a function of time at constant temperature $\left(25^{\circ} \mathrm{C}\right)$. Moreover, the samples were stored, at ambient temperature, for 24 hours, one week and one month. The stability of the formulations were also assessed visually.

One of the triterpene acid was incorporated to oil/ surfactant mixtures, homogenized with a vortex and placed in an ultrasound bath for $10 \mathrm{~min}$. The active solubility was determined by the sample observation using optical microscopy, the appearance of crystals has indicated exceeding the limit solubility of the acid). The nano-emulsions were formed by water addition to the oil/ surfactant/active mixtures, and stored in a water bath at $25^{\circ} \mathrm{C}$. The stability of the formulations were examined by visual and optical observation after 24 hours of the preparation.

\section{RESULTS AND DISCUSSION}

In the first stage of the studies different types of oils were tested to obtain stable nano-emulsion systems. All formulations were prepared by low energy emulsification method, by dropwise addition of water to the mixture of oil/surfactant in varied ratios 90:10, 80:10, 70:30, 60:40 and 50:50.

An area of the occurrence of stable nano-emulsions for each of the oils depended on the ratio of the oil/ surfactant. In case of Crodamol GTCC, transparent or transparent-bluish liquid dispersions appeared after addition of approximately 57 wt. \% of water for oil/surfactant (O/S) mixture of ratio 10:90, 20:80 and 30:70. Nano-emulsion region could not be extended at $\mathrm{O} / \mathrm{S}$ ratio higher than 40/60. For the Crodamol PC/Polysorbate $80 /$ water system, the region of nano-emulsions was determined for oil/surfactant $(\mathrm{O} / \mathrm{S})$ ratio 10:90 and $20: 80$, also after addition of approximately $57 \mathrm{wt}$. \% of water. The translucent emulsion area in case of oleic acid was identified, after addition of $80 \%$ of water phase to the mixture of oil/surfactant in a ratio $10: 90$ and 20:80. Table 1 shows the composition and characteristic of the systems.

\section{Size Distribution by Volume}

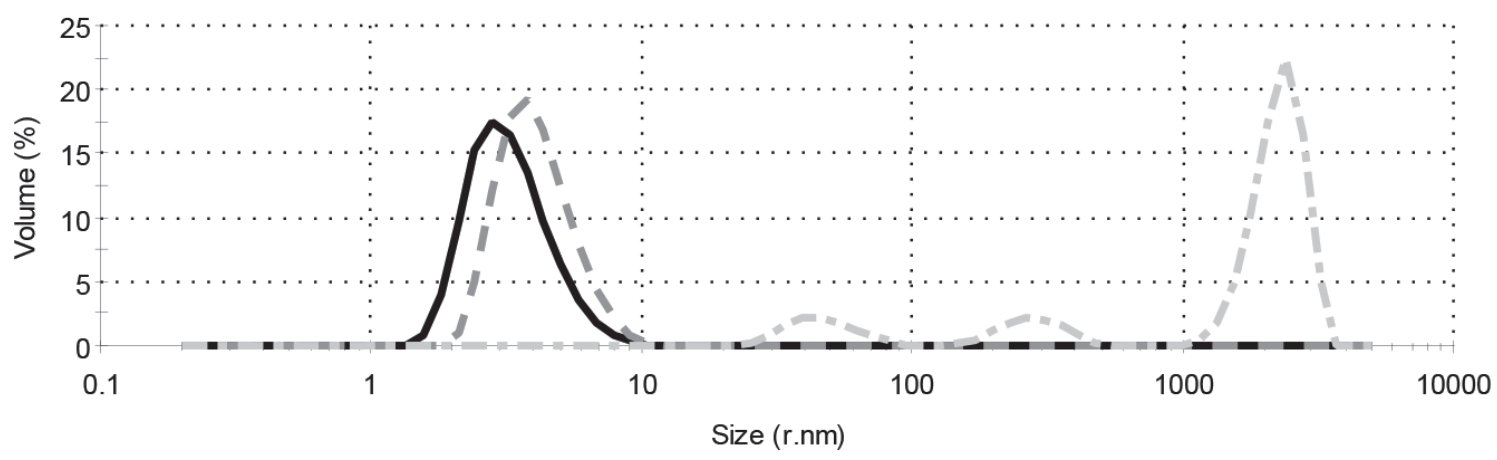

\begin{tabular}{|c|c|c|c|c|}
\hline & Record 225: NE-T80-GTCC-10:90 & -2. & & Record 226: NE-T80-PC-10:90 \\
\hline 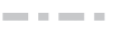 & Record 227: NE-T80-OA-10:90 & & & \\
\hline
\end{tabular}

Figure 1. Size distribution of the formulations based on different oils: NE-T80-GTCC-10:90 (Crodamol GTCC), NE-T80-PC-10:90 (Crodamol PC) and NE-T80-OA-10:90 (Oleic Acid). 


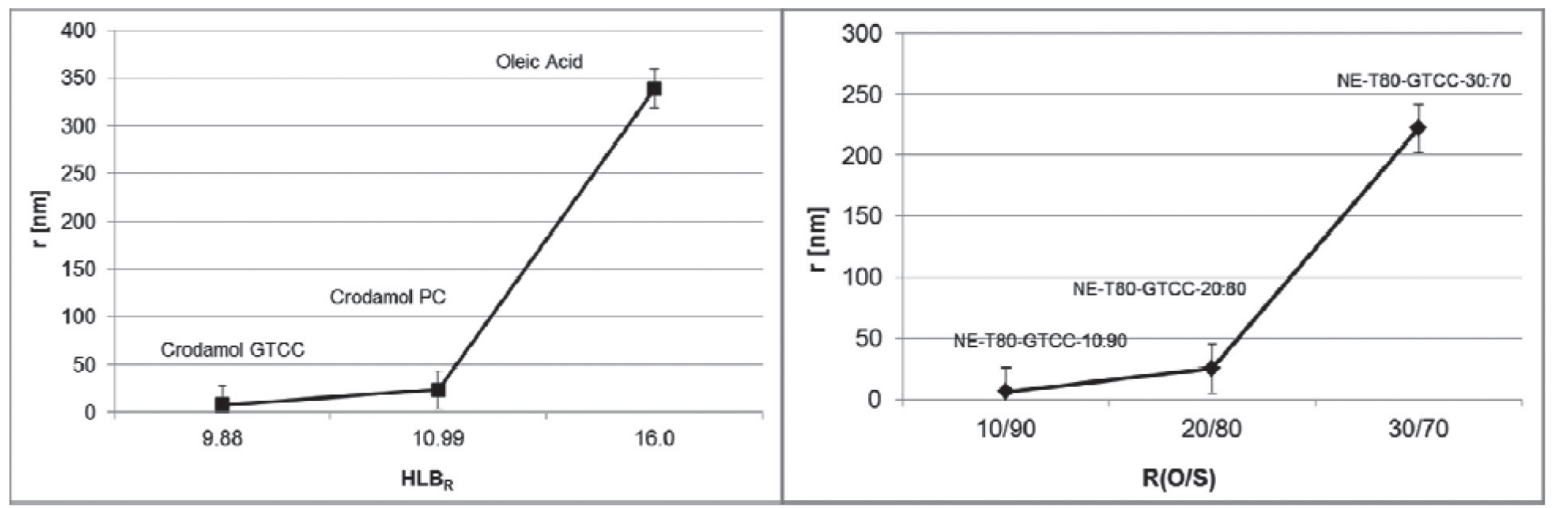

Figure 2. The droplet size dependence of hydrophilic-lipophilic balance $\left(H L B_{R}\right)$ of oils used in studies (a) and dependence of oil/surfactant ratio $\mathrm{R}(\mathrm{O} / \mathrm{S})$ for crodamol GTCC/Tween $80 /$ water system (b).

The droplet size of nano-emulsions was determined by means of DLS method. The obtained result showed that the droplet size of inert phase depend on kind of the oil. The date presented in Fig. 1 shows that formulation based on crodamol GTCC is characterized by the small droplets size $(\mathrm{r}=8 \mathrm{~nm})$, similarly to crodamol PC nano-emulsion $(\mathrm{r}=22 \mathrm{~nm})$ and contrary to oleic acidbased emulsion $(\mathrm{r}=339 \mathrm{~nm})$ at 10/90 oil/surfactant ratio. Moreover emulsion based on oleic acid is characterized by very high polydispersity. The difference between those two systems is very significant. It could be explain by the fact that caprylic/capric tiglicerydes are the most hydrophobic oil among the studied. It is characterized by the lowest value of required $\mathrm{HLB}_{\mathrm{r}}=9.88$, while the oleic acid is characterized by the highest $\mathrm{HLB}_{\mathrm{r}}=16.00$. It is believed that lipophilicity of oil phase significant influence on the size distribution and stability of nanoemulsions. (Fig. 2a).

Apart from the kind of the oil, also oil/surfactant ratio influences the properties of the nano-emulsion. Figure $2 \mathrm{~b}$ shows, on the bases of crodamol GTCC/ polysorbate 80 /water system, effect of $\mathrm{O} / \mathrm{S}$ ratio on the droplet size distribution. The data presented in Fig. $2 \mathrm{~b}$ indicates that the droplet size increases with the increase of $\mathrm{O} / \mathrm{S}$ ratio. For the oil/surfactant ratio in range from $10 / 90$ and $20 / 80$ the difference is quite small, mean radius of droplets is respectively: $r=8 \mathrm{~nm}$ and $\mathrm{r}=25$ $\mathrm{nm}$, while further increase in oil concentration causes significant changes in droplet size, in case of $\mathrm{O} / \mathrm{S}$ ratio

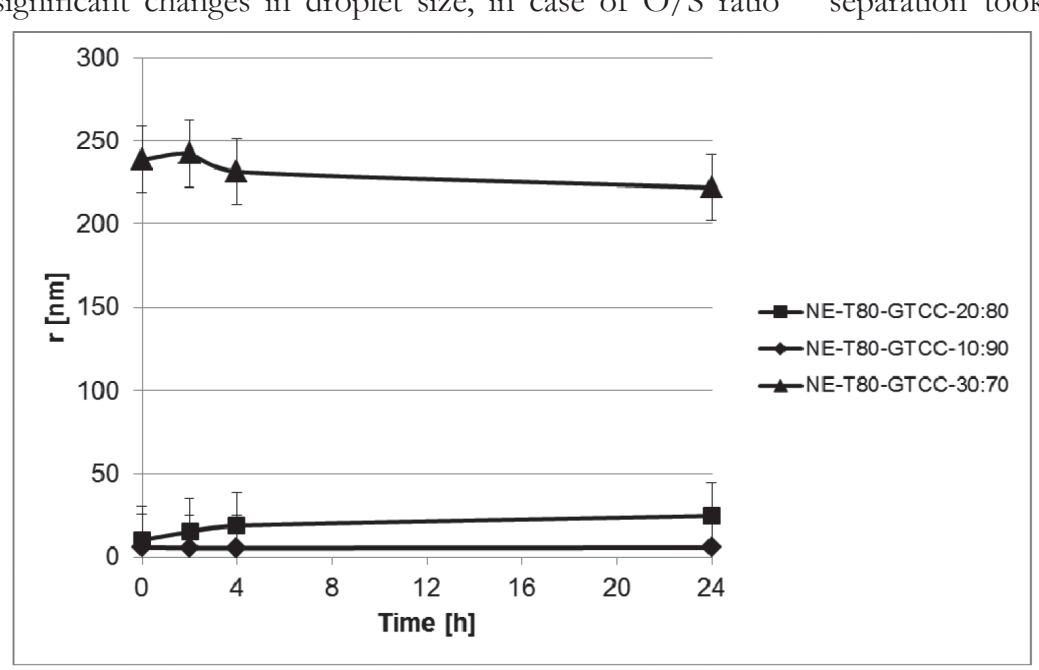

Figure 3. Stability of crodamol GTCC-based nano-emulsions.
$30 / 70$ the droplet size is $222 \mathrm{~nm}$. It should be noticed that nano-emulsion droplet sizes of water/non-ionic surfactant oil systems studied are expectionally small compared to those reported in the literature (Sadurni et al., 2005).

Effect of the incorporation of the active on nanoemulsion systems shows Table 1. Only in case of crodamol GTCC, stable, triterpene acid loaded nano-emulsion was obtained. In case of oleic acid and crodamol PC based systems crystallization effect of the active, at very low, (below therapeutic), concentrations were observed. The results are in agreement with literature data which show that medium-chain triglycerides are one of the effective solubilisers for many lipophilic actives incorporated in nano-emulsions e.g. carbamazepine (Kelmann et al., 2007), $\beta$-carotene (Yuan et al., 2008) or vitamin E (Saberi et al., 2013).

In the next stage of the work, the stability of nanoemulsions based on crodamol CTCC was determined by measuring droplet size dependence of time, at $25^{\circ} \mathrm{C}$. These systems were selected from the studied due to the best properties, i.e. lowest droplet size and low polidyspersity index (Table 1). The obtained results are showed in Fig. 3.

Crodamol GTCC-based nano-emulsions, irrespective of $\mathrm{O} / \mathrm{S}$ ratio, are stable systems in time. In all cases minor changes of droplet size distribution were observed within the 24 hours observation. In addition, no phase separation took place in this period of time (after one week and one month).

\section{CONCLUSIONS}

The obtained results showed that both, the kind of the oil phase and the $\mathrm{O} / \mathrm{S}$ ratio influenced on the nano-emulsion properties. Systems based on crodamol GTCC, are very stable formulations, characterizing by the smallest droplet size The droplet size decreases when the surfactant concentration increases in emulsion system.

Additionally, crodamol GTCC/ Polisorbate 80 nano-emulsions, due to their good solubility of triterpenes, no crystallization effect of the active was observed), could be used as the self-emulsifying triterpenoic 
acid delivery systems, suitable for cosmeceutical applications.

\section{Acknowledgements}

The research (work) was supported by the European Union through the research scholarship "Doctus Małopolski fundusz stypendialny dla doktorantów”.

\section{REFERENCES}

Gao X-H, Zhang L, Wei H, Chen H-D (2008) Efficacy and safety of innovative cosmeceuticals. Clin Dermatol 26: 367-374.

El Maghraby GM (2008) Transdermal delivery of hydrocortisone from eucalyptus oil microemulsion: effects of cosurfactants. Int J Pharmaceut 355: 285-292.

Peltola S, Saarinen-Savolainen P, Kiesvaara J, Suhonen TM, Urtii A (2003) Microemulsions for topical delivery of estradiol. Int J Pharmaceut 254: 99-107.

Teo B, Basri M, Zakaria M, Salleh A, Rahman R, Rahman M (2010) A potential tocopherol acetate loaded palm oil esters-in-water nanoemulsions for nanocosmeceuticals. J Nanobiotechnol 8: 4.

Tadros T, Izquierdo P, Esquena J, Solans C (2004) Formation and stability of nano-emulsions. Adv Colloid Interface Sci 108-109: 303-318.

Gursoy RN, Benita S (2004) Self-emulsifying drug delivery systems (SEDDS) for improved oral delivery of lipophilic drugs. Biomedicine ¿ Pharmacotherapy 58: 173-182.
Hong J-Y, Kim J-K, Song Y-K, Park J-S, Kim C-K (2006) A new selfemulsifying formulation of itraconazole with improved dissolution and oral absorption. J Controlled Release 110: 332-338.

Balakrishnan P, Lee B-J, D. Oh DH, Kim JO, Lee Y-I,. Kim D-DJee J-P, Lee Y-B, Woo JS, Yong ChS, Choi H-G (2009) Enhanced oral bioavailability of Coenzyme Q10 by self-emulsifying drug delivery systems. Int J Pharmaceut 374: 66-72.

Rao S, Agarwal P, Shao J (2008) Self-nanoemulsifying drug delivery systems (SNEDDS) for oral delivery of protein drugs. Int J Pharmaceut 362: 10-15.

Wang G, Tang W, Bidigare RR (2005) Terpenoids as Therapeutic Drugs and Pharmaceutical Agents in Natural Products: Drug Discovery and Therapeutic Medicine, pp 97-227. Humana Press Inc., Totowa, NJ.

Silva M, David J, Silva L, Santos R, David J, Lima L, Reis P, Fontana $\mathrm{R}$ (2012) Bioactive oleanane, lupane and ursane triterpene acid derivatives. Molecules 17: 12197-12205.

Sadurni N, Solans C, Azemar N, Garcia-Celma MJ (2005) Eur J Pharmaceut Sci 26: 438-445.

Kelmann R, Kuminek G, Teixeira H, Koester L (2007) Carbamazepine parenteral nanoemulsions prepared by spontaneous emulsification process. Inte I Pharmaceut 342: 231-239.

Yuan Y, Gao Y, Zhao J, Mao L (2008) Characterization and stability evaluation of b-carotene nanoemulsions prepared by high pressure homogenization under various emulsifying conditions. Food Res Int 41: 61-68.

Saberi Ah, Fang Y, McClements FD (2013) Fabrication of vitamin Eenriched nanoemulsions: Factors affecting particle size using spontaneous emulsification. J Colloid Interface Sci 391: 95-102. 